\title{
Lufenuron: A Potential Chitin Synthesis Inhibitor Against Aedes aegypti L.
}

\author{
Kungreiliu Panmei ${ }^{1 *}$, P Lanbiliu ${ }^{1}$, Roopa Rani Samal ${ }^{1}$, Sarita Kumar ${ }^{1}$ \\ ${ }^{1}$ Acharya Narendra Dev College (University of Delhi), Govindpuri, New Delhi-110019, India \\ "Corresponding author. Email: kungreiliu@gmail.com
}

\begin{abstract}
Chemical control of dengue vector, Aedes aegypti is impaired due to development of resistance to conventional insecticides. Insect Growth Regulators (IGRs) are considered more suitable and effective vector control agents as they specifically inhibit chitin biosynthesis, a process absent in vertebrates, and impose less adverse effects on beneficial insects and the environment. Present study investigates Lufenuron, a Chitin Synthesis Inhibitor (CSI), as a control agent of Ae. aegypti. Different instars of Ae. aegypti were exposed to a range of concentrations of Lufenuron as per WHO protocol. The investigations showed the effective hormone-mimetic effect of Lufenuron resulting in the formation of a significant number of larval-pupal and pupal-adult intermediates with the maximum number observed on exposure to L3 ( $\mathrm{L}-\mathrm{P}=17 \%, \mathrm{P}-\mathrm{A}=21 \%$ ). Approximately $20 \%$ of L2 instars either could not moult and remained trapped inside the new exuviae or possessed bulged abdomen while some showed ruptured exoskeleton. The results showed increase in $\mathrm{IE}_{30}$ from L1 $(0.00010 \mathrm{ppm})$ to L4 stage $(0.00013 \mathrm{ppm})$; the L2 stage exhibiting maximum $\mathrm{IE}_{30}(0.00025 \mathrm{ppm})$. The median emergence suppression $\left(\mathrm{IE}_{50}\right)$ doses of the Lufenuron were found to be $0.00057 \mathrm{ppm}$ for L1, $0.00047 \mathrm{ppm}$ for L2, $0.00050 \mathrm{ppm}$ for L3 and $0.00096 \mathrm{ppm}$ for L4. The results also revealed increased duration of larval development and inability of pupae to develop into adults, as compared to the controls. The investigations indicate the potential use of Lufenuron as the control agent of Ae. aegypti. Further research is being conducted to understand its mode of action to develop effective control strategies.
\end{abstract}

Keywords: Aedes aegypti, growth inhibition, intermediates, Lufenuron, hormone-mimetic

\section{INTRODUCTION}

Dengue, yellow fever and Chikungunya are the most prevailing diseases worldwide, responsible for millions of deaths each year [1]. Aedes aegypti has been the most devastating vector in last few decades and plays the major vector in spreading these diseases [2]. The World Health Organization has reported a global estimate of 3.9 billion people inhabiting 128 countries facing risk of dengue infection while 60 countries were identified as the Chikungunya-prone areas by WHO [3]. The dengue/dengue haemorrhagic fever data recorded by NVBDCP (National Vector Borne Disease Control Programme), India showed a total of 1,29,166 cases and 245 deaths in the year 2016 which shot up to $1,88,401$ cases and 325 deaths in 2017. In November 2018, though a decline to 89,974 cases and 144 deaths was observed; the total data is yet to be reported [4].

Primary control measures of the mosquito vectors have mainly been focused on the use of insecticides, primarily those which target the insect central nervous system. Nevertheless, the indiscriminate use and rising reports of resistance development in the vector population to these insecticides has led to the consequent failures of control efforts [5]. Thus, new and effective tools have to be taken into consideration and identified for the control of these important disease vectors.

A recent approach to insect pest control is the use of substance with distinct mechanism of action. Substances like Insect Growth Regulators (IGRs) have a selective mode of action as compared to other synthetic insecticides. Synthetic insecticides; like organophosphates and carbamates; interfere with various physiological processes of insects, while IGRs act on the target species by adversely affecting their growth and development leading to developmental abnormalities impairing the survival of insect [6, 7]. Apart from this, these compounds are considered safer to the non-target organisms than conventional insecticides [8].

Based on the mode of action, IGRs can be grouped into two types: chitin synthesis inhibitor (CSI) and substances that interfere with the action of insect hormones [6]. CSIs are benzoylphenyl urea compounds and were first discovered in the 1970s [9]; they can affect reproduction and development of organisms to varying degrees. Insect larvae treated with CSIs fail to ecdyse because of the inhibition of cuticle synthesis even if moulting occurs [6]. These compounds can also prevent the formation of adequate peritrophic membrane (PM) lining the insect gut [10], which consequently disturbs the 
normal functioning, leaving the insect more susceptible to exogenous compounds [11]. Adults emerging from CSIexposed larvae can have physiological constraints leading to diminished physical and reproductive fitness [12]. The effect of CSIs can also depend on the variety of insect species, the developmental stage at the time of application, the dose administered and the kind of compound used [13, 14].

Present study investigates the growth inhibitory and growth regulatory potential of Lufenuron, a CSI, against Ae. aegypti population.

\subsection{Materials and Methods}

\subsubsection{Mosquito rearing}

A culture of Ae. aegypti was maintained at $28 \pm 1{ }^{\circ} \mathrm{C}$, $80 \pm 5 \%$ relative humidity (RH) and 14:10 L:D photoperiod [15]. Adults were reared in a cloth cage and fed on deseeded raisins soaked in water; a source of food primarily for the male adults. Female adults were given periodic blood meals for egg maturation. Enamel bowls; lined by Whatman filter strips and half-filled with dechlorinated water; were used to collect the eggs. The filter strips laden with eggs were transferred to an enamel tray containing dechlorinated water; for hatching. Larvae were fed daily with food; containing combination of live active yeast and dog biscuit (1:3). The pupae formed were transferred into the cloth cage for adult emergence.

\subsubsection{Emergence inhibition bioassay}

Lufenuron, a Chitin Synthesis Inhibitor (CSI) with $\geq$ $98.0 \%$ purity, was procured from M/s Sigma-Aldrich. The compound was weighed and diluted in ethanol obtained from eMerck. The stock solution of $1 \%$ was prepared and stored in the refrigerator at $4{ }^{\circ} \mathrm{C}$ for future use.

The emergence inhibition bioassay of Lufenuron was conducted against different larval stages (L1, L2, L3, L4) of Ae. aegypti as per the WHO recommended protocol with slight modifications [16]. A graded series of Lufenuron was prepared using ethanol as the solvent. Batches of 20 instars of each stage; L1, L2, L3 and L4; were taken in a plastic bowl containing $99 \mathrm{ml}$ of distilled water and transferred into a glass jar containing $100 \mathrm{ml}$ distilled water and $1 \mathrm{ml}$ of a particular concentration of Lufenuron. Three replicates were performed for each dilution. Controls were run simultaneously using only the diluting solvent, ethanol. Due to the longer duration of the assay, both the treated and control larvae were fed periodically. After a $24 \mathrm{hr}$ exposure period, the larvae and pupae formed were transferred to a glass jar containing dechlorinated water. Mortality was recorded until adult emergence. Moribund larvae, dead larvae and pupae, and the incompletely emerged adult mosquitoes; not completely separated from the pupal case, were counted as "affected". Number of empty pupal cases were scored as the successfully emerged adults. The experiment was concluded when all the larvae or pupae in the controls developed and emerged as adults.

\subsubsection{Statistical analysis of data}

If adult emergence in the control was less than $80 \%$, the test was discarded and repeated. Where the percentage is between $80 \%$ and $95 \%$, the data are corrected using Abbott's formula [17]. The overall emergence of adults reflects growth inhibitory activity of Lufenuron against $A e$. aegypti. Percent emergence inhibition (IE\%) was calculated using the formula:

$$
\mathrm{IE} \%=100-\left\{\frac{T \times \mathbf{1 0 0}}{C}\right\}
$$

Where, $\mathrm{T}=$ percentage of survival or emergence in treated batches, and $\mathrm{C}=$ percentage of survival or emergence in control batch.

The data was subjected to regression analysis using computerized SPSS 22.0 Programme. The $\mathrm{IE}_{30}$ and $\mathrm{IE}_{50}$ values with $95 \%$ fiducial limits were calculated in each bioassay to measure the variation between the test samples. During each emergence inhibition bioassay, dead larvae of Ae. aegypti in both the control and experimental setups were scrutinized under light microscope for behavioural and morphological modifications, if any. Behavioural changes like excitation, restlessness, sluggishness, etc., were recorded, if observed. Changes in the body segments like the head, thorax and abdomen and organs such as the antennae, eyes, setae, siphon and anal fins were photographed and compared with controls.

\subsection{Our Contribution}

This study shows the potential growth inhibitory effects of Lufenuron on different stages of mosquito larvae. It variably affects the various phases of the target insect's life cycle, consequently disrupting the continuance of next generation. As insects are not expected to develop resistance to IGRs and these compounds may even be effective against those insects which have developed resistance to conventional insecticides; the use of Lufenuron to manage mosquito population can be an effective and sustainable strategy.

\subsection{Paper Structure}

The rest of the paper is organized as follows. Section 2 describes the impact of Lufenuron on the growth and development of Ae. aegypti. The results obtained are analysed using suitable statistical tools and are discussed in comparison to similar experiments performed previously using different compounds with similar modes 
of action. Section 3 concludes the paper stating the possible use of Lufenuron in mosquito management. The recommendations are made and direction for further research are presented.

\section{RESULTS AND DISCUSSION}

The result of the inhibition of emergence bioassay performed on the first, second, third and fourth instars of Ae. aegypti with CSI, Lufenuron are presented in Table 1. Mortality in each larval stage was recorded up to adult emergence. Mortality and deformity of larva because of the failure to shed their cuticle was observed as a common trend [Figure 1a]. Similar reports were given by Aziz [18] on application of three different IGRs on Ae. aegypti early $4^{\text {th }}$ instars and Arora [19] who exposed Tribolium castaneum to Lufenuron. It was also observed that because of premature apolysis, larvae remained trapped in exuviae, did not moult completely and died slowly due to starvation and desiccation. Such manifestations were also noted on treating Culex pipiens with another CSI, Novaluron [8]. High larval mortality relative to pupa and adult observed on larval exposure to higher Lufenuron doses; pointing to the direct relationship between CSI concentrations and the precocity of their effects; were in agreement with observations made by some authors [20-22].

The investigations also showed the effective hormonemimetic effect of Lufenuron on Ae. aegypti resulting in the formation of a significant number of larval-pupal (Figure
$1 \mathrm{~b}, \mathrm{c}, \mathrm{d})$ and pupal-adult intermediates during development (Figure 2e, f). The maximum number of intermediates were observed on exposure of L3 to Lufenuron ( $\mathrm{L}-\mathrm{P}=17 \%$, $\mathrm{P}-\mathrm{A}=21 \%)$. It suggests the significant potential of Lufenuron to influence mosquito reproduction by hormonal imbalance as is seen in the cases of $\mathrm{JH}$ analogues [23, 24]. Reports have shown the development of abnormal pupal-adult intermediates on exposure of $T$. castaneum to Lufenuron [19]. A number of pupae retained the larval exuviae on a portion of the abdomen on treating Ae. aegypti larvae with pyriproxyfen and diflubenzuron [25]. This research also observed that successfully moulted larvae reached the adult stage but remain trapped with appendages and wings in the pupal exuviae (Figure 1e, f). This is in agreement with the observations by other investigators, who found that mosquito adults were incompletely emerged with the legs and tarsi attached to the pupal exuviae on treatment with pyriproxyfen and diflubenzuron $[25,26]$.

The investigation clearly indicates the emergence suppression potential of Lufenuron against adult mosquitoes with $\mathrm{IE}_{30}$ values of $0.00010 \mathrm{ppm}, 0.00025$ ppm, $0.00012 \mathrm{ppm}$ and $0.00013 \mathrm{ppm}$ in L1, L2, L3 and L4 stages, respectively. The $\mathrm{IE}_{50}$ value was least for the $\mathrm{L} 2$ stage $(0.00047 \mathrm{ppm})$ while highest for L4 stage $(0.00096$ ppm). Studies by Aziz [18] showed the emergence inhibition potential of three IGRs against early $4^{\text {th }}$ instars of Ae. aegypti, Sumilarv $0.5 \mathrm{G}$ being the most effective $\left(\mathrm{IC}_{50}=0.0004 \mathrm{ppm}\right)$, followed by Diflox Flowable $\left(\mathrm{IC}_{50}=\right.$ $0.002 \mathrm{ppm})$ and Baycidal $25 \mathrm{wp}\left(\mathrm{IC}_{50}=0.047\right)$. The results

Table 1 Emergence Inhibition (IE) bioassay with Lufenuron against $1^{\text {st }}, 2^{\text {nd }}, 3^{\text {rd }}$ and $4^{\text {th }}$ instars larvae of Aedes aegypti

\begin{tabular}{|c|c|c|c|c|c|}
\hline $\begin{array}{l}\text { Larval } \\
\text { Instars }\end{array}$ & $\begin{array}{c}\mathrm{IE}_{30} \\
\text { (95\% Fiducial } \\
\text { limits) }\end{array}$ & $\begin{array}{c}\text { IE }_{50} \\
\text { (95\% Fiducial } \\
\text { limits) }\end{array}$ & $\chi^{2}$ & RC & S.E. \\
\hline L1 & $\begin{array}{c}0.00010 \\
(0.00004-0.00019)\end{array}$ & $\begin{array}{c}0.00057 \\
(0.00036-0.00083)\end{array}$ & 1.719 & 0.69959 & 6.62395 \\
\hline L2 & $\begin{array}{c}0.00025 \\
(0.00018- \\
0.00031)\end{array}$ & $\begin{array}{c}0.00047 \\
(0.00038- \\
0.00056)\end{array}$ & 3.450 & 1.85663 & 12.53597 \\
\hline L3 & $\begin{array}{c}0.00012 \\
(0.00005- \\
0.00019)\end{array}$ & $\begin{array}{c}0.00050 \\
(0.00033- \\
0.00069)\end{array}$ & 5.671 & 0.84087 & 7.79202 \\
\hline L4 & $\begin{array}{c}0.00013 \\
(0.00004- \\
0.00024)\end{array}$ & $\begin{array}{c}0.00096 \\
(0.00061- \\
0.00153)\end{array}$ & 3.741 & 0.59700 & 5.72142 \\
\hline
\end{tabular}

$\mathrm{IE}_{30}=$ concentration that inhibits $30 \%$ of adult emergence; $\mathrm{IE}_{50}=$ concentration that inhibits $50 \%$ of adult emergence; $\chi^{2}=$ chi square; $\mathrm{RC}=$ Regression Coefficient; S.E.= Standard error; test samples were transformed into $\log \operatorname{covariant}(\log 10)$; $\mathrm{p}<0.05$ level of significance; values are mean of three replicates. 
slow acting as compared to the neurotoxic insecticides. Some larvae also exhibited toxic symptoms like tremors and convulsions settling at the bottom of the glass jar followed by paralysis and stiffness. Damage to the breathing siphon could be the reason for alteration and reduction in the motility of the larvae, preventing it from rising to the surface. The absorption process by anal papillae was interrupted due to damage caused by Lufenuron. It has been reported earlier that anal papillae of mosquito larvae exhibit high cellular activity due to numerous mitochondria, and absorb chlorine, sodium, and potassium from the water to maintain the normal ionic composition of the haemolymph [30].

These studies indicate that Lufenuron, even at very low concentrations, can alter the behaviour and morphology of Ae. aegypti with other possible physiological alterations and thus, can arrest their development. As is evident from the results, it is effective against all the life stages of Ae. aegypti; all four stages of larvae, pupae and adult.

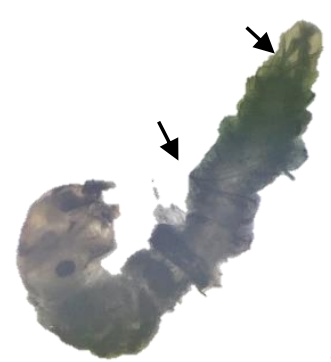

a

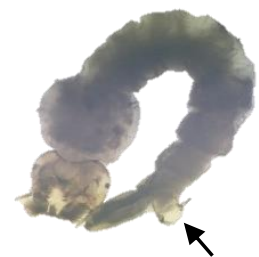

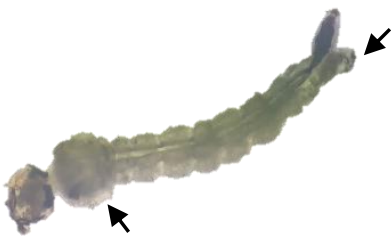

b

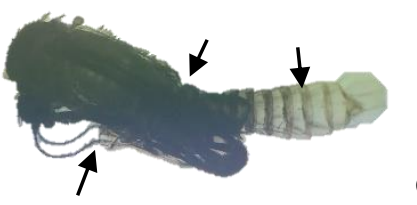

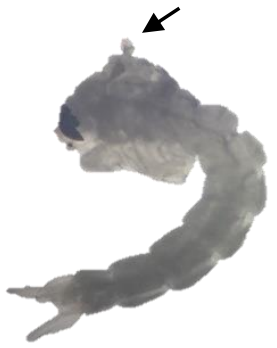

c

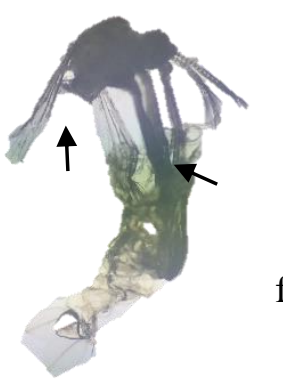

Figure 1 Larval-pupal and pupal-adult intermediates formed in Aedes aegypti on larval exposure to Lufenuron (Represented by arrows) : (a) Larva trapped in old exuviae; affected respiratory siphon, (b) Visible pupal horn in larvalpupal intermediate; anal papillae absent, (c) Larval-pupal intermediate with pupal horn, (d) Anal papillae absent, (e) Pupal-adult intermediate; antennae, wings and legs trapped in pupal exuviae, (f) Adult with trapped legs; abdomen region lost 


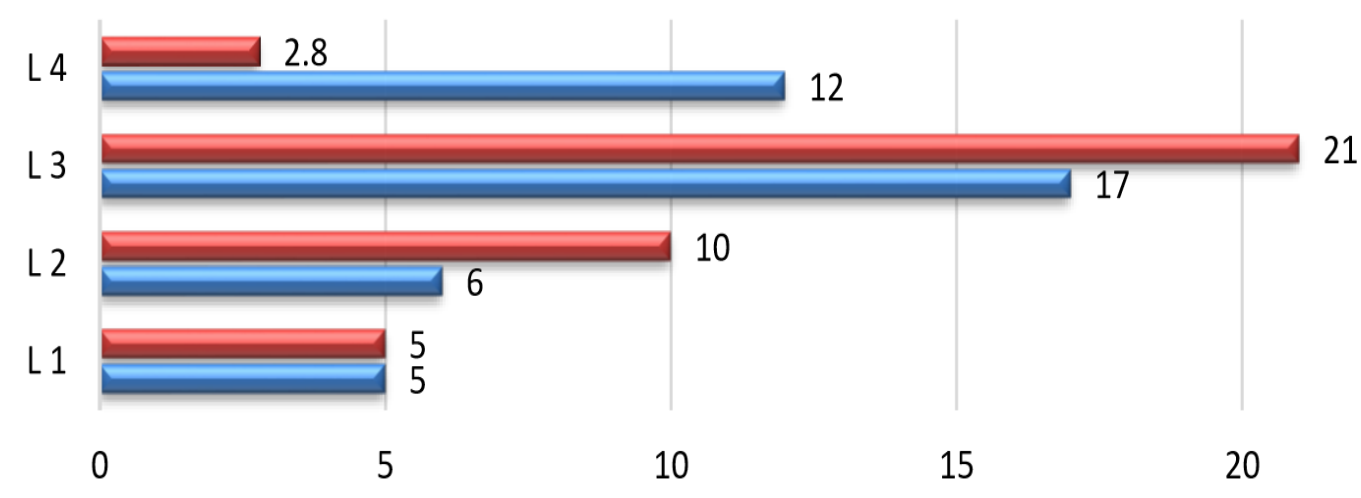

$\square P-A \square L-P$

Figure 2 Percent intermediates formed in Aedes aegypti on exposure of different instars to Lufenuron. $\mathrm{L} 1=1^{\text {st }}$ larval instar; L2 $=2^{\text {nd }}$ larval instar; L3 $=3^{\text {rd }}$ larval instar; $\mathrm{L} 4=4^{\text {th }}$ larval instar; $\mathrm{L}-\mathrm{P}=$ larval-pupal intermediate; $\mathrm{P}-\mathrm{A}=$ pupal-adult intermediate

\section{CONCLUSION}

The outcome results showed the efficacy and latent effects of Lufenuron on different stages of Ae. aegypti mosquito with behavioural, physiological and morphological alterations. The use of IGRs as an alternative or integrated solution to other methods of mosquito control, such as chemical insecticide, could enhance the efficiency of control operations by reducing the risk of developing resistance to the same process. The possible use of Lufenuron as one of the components in vector control programme can be an effective and sustainable strategy for management of Ae. aegypti.

\section{ACKNOWLEDGMENT}

Authors thankfully acknowledge Council of Scientific and Industrial Research (CSIR), New Delhi for their financial assistance. The authors are also grateful to the Principal, Acharya Narendra Dev College, University of Delhi for making laboratory and culture facilities available for conducting the experiments.

\section{REFERENCES}

[1] S. Salokhe, S. Mukherjee, S. Deshpande, V. Ghule, J. Mathad, Effect of sub-lethal concentrations of insect growth regulator, lufenuron on larval growth and development of Aedes aegypti, Curr. Sci. 99 (9) (2010) 1256-1259.

[2] T. Yang, L. Liang, F. Guiming, S. Zhong, G. Dong, R. Xu, G. Zhu, N. Shi, F. Fan, Q. Liu,
Epidemiology and vector efficacy during dengue fever outbreak in Cixi, Zhejiang province, China, J. Vector Ecol. 34 (1) (2009) 148-154. DOI: https://doi.org/10.1111/j.1948-7134.2009.00018.x

[3] World Health Organisation, Dengue and severe dengue factsheet, https://www.who.int/news-room/fact-sheets.

[4] National Vector Borne Disease Control Programme (NVBDCP) Dengue Cases and Deaths in the Country since 2015 (2018). http://nvbdcp.gov.in/ index4.php?lang $=1 \&$ level $=0 \&$ linkid $=431 \&$ lid $=37$ 15

[5] M. Zaim, P. Guillet, Alternative insecticide: an urgent need, Trends Parasitol. 18 (4) (2002) 161163. DOI: https://doi.org/10.1016/S14714922(01)02220-6

[6] H. Tunaz, Insect growth regulators for insect pest control, Turk. J. Agric. For. 28 (6) (2014) $377-$ 387.

[7] J.B. Siddall, Insect growth regulators and insect control: a critical appraisal, Environ. Health Perspect. 14 (1976) 119-26. DOI: https://doi.org/10.1289/ ehp.7614119

[8] N.E.H. Djeghader, L. Aïssaoui, K. Amira, H. Boudjelida, Impact of a chitin synthesis inhibitor, Novaluron, on the development and the reproductive performance of mosquito Culex pipiens, World Appl. Sci. J. 29 (7) (2014) 954960. DOI: 10.5829/idosi.wasj.2014.29.07.82190 
[19] V. Arora, Effect of sublethal concentrations of lufenuron on growth, development and reproductive performance of Tribolium castaneum (Herbst) (Coleoptera : tenebrionidae ), Int. J. Appl. Biol. Pharm. 3 (2012) 111-122.

[20] N.G. Fontoura, D.F. Bellinato, D. Valle, J.B.P. Lima, The efficacy of a chitin synthesis inhibitor against field populations of organophosphateresistant Aedes aegypti in Brazil, Mem. Inst. Oswaldo Cruz. 107 (3) (2012) 387-395. DOI: http://dx.doi.org/ 10.1590/S0074-02762012 0 00300014

[11] P. Wang, R.R. Granados, Calcofluor disrupts the midgut defense system in insects, Insect Biochem. Mol. Biol. 30 (2) (2000) 135-143. DOI: https://doi.org/10. 1016/S0965-1748(99)00108-3

[12] K. Mondal, S. Parween, Insect growth regulators and their potential in the management of storedproduct pests, Integ. Pest Manag. Rev. 5 (4) (2000) 255-295. DOI: https://doi.org/10.1023/ A:1012901832162

[13] V. Vasuki, A.R. Rajavel, Influence of short time exposure to an insect growth regulator, hexaflumuron, on mortality and adult emergence of vector mosquitoes, Mem. Inst. Oswaldo Cruz. 87 (2) (1992) 275-283. DOI: http://dx.d oi.org/10.1590/S0074-02761992000200016

[14] T.G. Wilson, J.R. Cryan, Lufenuron, a chitinsynthesis inhibitor, interrupts development of Drosophila melanogaster, J. Exp. Zool. 278 (1) (1997) 37-44. DOI: https://doi.org/10.1002/(SICI)1097010X(19970501)278:1<37::AID-JEZ4>3.0.CO;27

[15] R. Warikoo, S. Kumar, Impact of Argemone mexicana extracts on the cidal, morphological, and behavioural response of dengue vector, Aedes aegypti L. (Diptera: Culicidae), Parasitol. Res. 112 (10) (2013) 3477-3484. DOI: https://doi.org/10.1007/s004 36-013-3528-7

[16] World Health Organization, Guidelines for laboratory and field testing of mosquito larvicides, (2005).

Https://apps.who.int/iris/handle/10665/69101

[17] W.B. Abbott, A method for computing the effectiveness of an insecticide, J. Econ. Entomol. 18 (2) (1925) 265-267.

[18] A.T. Aziz, Insecticidal activity of three insect growth regulators towards the dengue and Zika virus vector Aedes aegypti in Saudi Arabia, J. Entomol. Zool. Stud. 5 (2017) 961-966.

[21] M.S. Mulla, U. Thavara, A. Tawatsin, J. Chompoosri, M. Zaim, T. Su, Laboratory and field evaluation of novaluron, a new acylurea insect growth regulator, against Aedes aegypti (Diptera: Culicidae), J. Vector. Ecol. 28 (2003) 241-254.

[22] T. Su, M.S. Mulla, M. Zaim, Laboratory and field evaluations of novaluron, a new insect growth regulator (IGR), against Culex mosquitoes, J. Am. Mosq. Contrl. Assoc. 19 (4) (2003) 408-418.

[23] D. L. Bull, Toxicity and pharmacodynamics of avermectin in the tobacco budworm, corn earworm and fall armyworm (Lepidoptera: Noctuidae), J. Agric. Food Chem. 34 (1) (1986) 74-78. DOI: https://doi.org/10.1021/jf00067a021

[24] D.C. Deecher, J. Brezner, S.W. Tanebaum, Sublethal effects of avermectin and milbemycin on gypsy moth (Lepidoptera: Lymantriidae), J. Econ. Entomol. 83 (3) (1990) 710-714. DOI: https://doi.org/10.1093/jee/83.3.710

[25] H. Kamal, E. Khater, The biological effects of the insect growth regulators; pyriproxyfen and diflubenzuron on the mosquito Aedes aegypti, J. Egyptian Soc. Parasitol. 40 (3) (2010) 565-574.

[26] A.C. Bridges, J.K. Ulsan, R.T. Mayer, Effects of a new fluorescent insects growth regulators on the larval insects of Aedes aegypti, Mosq. News 37 (1977) 227-33

[27] A.J. Martins, T.A. Belinato, J.B.P. Lima, D. Valle, Chitin synthesis inhibitor effect on Aedes aegypti populations susceptible and resistant to the organophosphate temephos, Pest Manag. Sci. 64(6) (2008) 676-680. DOI: https://doi.org/10.1002/ps.1547

[28] T.A. Belinato, A.J. Martins, J.B. Pereira Lima, D. Valle, Effect of triflumuron, a chitin synthesis inhibitor, on Aedes aegypti, Aedes albopictus and Culex quinquefasciatus under laboratory conditions. Parasites \& Vectors 6(1) (2013) 83. DOI: https://doi.org/10. 1186/1756-3305-6-83 
[29] C.P. Batra, P.K. Mittal, T. Adak , M.A. Ansari, Efficacy of IGR compound Starycide 480 SC (Triflumuron) against mosquito larvae in clear and polluted water, J. Vector Borne Dis. 42 (3) (2005) 109-116.

[30] A. Montaño-Reyes, C. Llanderal-Cázares, J.M. Valdez-Carrasco, K. Miranda-Perkins, H. Sánchez-Arroyo, Susceptibility and alterations by diflubenzuron in larvae of Aedes aegypti, Arch. Insect Biochem. Physiol. 102 (2) (2019) e21604. DOI: $10.1002 / \operatorname{arch} .21604$ 